\title{
Nursing Challenges Continue Into 2022
}

As the New Year begins, I would like to thank the leadership of the boards of nursing and other national nursing organizations for their collegiality and comradery during the past 2 years as we addressed several difficult challenges related to COVID-19. Together, we produced policy briefs/statements that tackled issues related to clinical experiences during COVID-19 (Academic-Practice Partnerships), COVID-19 vaccine administration, clinical experiences for unvaccinated nursing students, and the dissemination of nonscientific and misleading COVID-19 information by nurses. The National Council of State Boards of Nursing (NCSBN) very much appreciates its partners' insight, support, and willingness to take a stance on these tough issues.

Accompanying this issue of the Journal of Nursing Regulation is NCSBN's annual environmental scan. The report describes the current state of healthcare, education, and regulation and provides implications for the year ahead. Despite all that has happened to change the world since 2020, the underlying message of this year's environmental scan is resilience. The nursing workforce, educators, and regulators have experienced the worst healthcare crisis in more than a century, and yet, our report is marked by achievement and innovation.

I hope you will take time to review the environmental scan. It outlines the achievements we will be building upon over the course of the next few years and provides insights into potential challenges we may face as we move forward together. Based on the report, here are several expectations for the year ahead:

- As COVID-19 continues, we can anticipate further disruption to the front-line nursing workforce as hospitals and other institutions compete for staff. There is a greater need than ever for states to adopt the Nurse Licensure Compact and APRN Compacts to ensure maximum mobility and flexibility for nurses and employers.

- Employers will be addressing workforce needs and assessing workforce environments. Expect a greater shift to remote working in many non-front-line positions.
- There will continue to be a shift toward online/remote education. Although remote education will be advantageous for many nursing students, regulators need heightened awareness of individuals and companies that may capital-

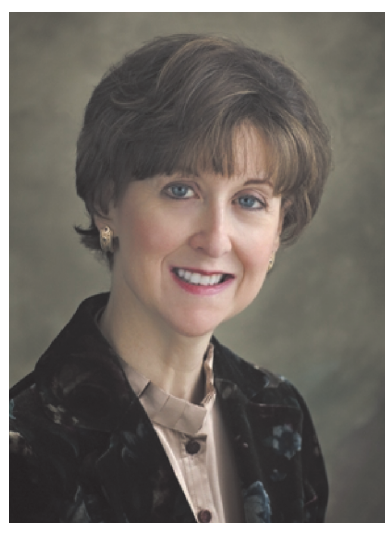
ize on these opportunities to open fraudulent, non-board-approved programs.

- Two issues need to be a focus for nursing education in 2022 and beyond. First, educators must teach the importance of the social determinants of health and evidence-based practice so that nurses can address the complex aspects of patient care that are part of the social determinants of health. Second, educators must emphasize science and focus on health literacy throughout the nursing program.

- Vaccine hesitancy and misinformation about COVID-19, masking, and vaccines will continue to be a major challenge for the nursing profession in 2022.

- There continues to be a fluctuation in the numbers of LPNs and LPN programs and a shift in practice facilities from long-term care to acute care facilities. This shift must be monitored, as public safety will be at risk if staffing at long-term care facilities suffers.

- Regulators, educators, and practitioners must continue to address the challenges of implicit bias and systemic racism.

- Expect technology and robotics to continue to advance and become routine aspects of nursing care. Regulators need to be aware of these advances and their impact on public safety.

Thank you for your readership throughout the years. My best wishes for a happy and safe New Year!

Maryann Alexander, PhD, RN, FAAN

Editor-in-Chief 